\title{
CORRESPONDENCE
}

\section{A Rare Case of Roseomonas gilardii Peritonitis in a Patient on Continuous Ambulatory Peritoneal Dialysis}

\section{Editor:}

There have been only 5 reported cases of Roseomonas peritonitis in peritoneal dialysis (PD) patients (1-4). Our 61-year-old patient on continuous ambulatory PD (CAPD) presented with asymptomatic, cloudy peritoneal dialysate. Intraperitoneal (IP) cloxacillin and ceftazidime was initiated. Blood test results were as follows: C-reactive protein $168 \mathrm{mg} / \mathrm{L}$, white cells $15.4 \times 10^{9} / \mathrm{L}$. Dialysate cell count was 40 cells/ $\mathrm{mm}^{3}$, predominantly polymorphs. Dialysate fluid cleared up on day 3 , with corresponding negative cell counts. Peritoneal dialysate culture grew Roseomonas gilardii on day 6 , resistant to ceftazidime, cefeperazone and piperacillin, and sensitive to amikacin, gentamycin, imipenem, ciprofloxacin, cefepime, and meropenem. Clinical improvement led to continuation of IP ceftazidime and cloxacillin. Two days later, dialysate cell counts rebounded to 180 cells $/ \mathrm{mm}^{3}$. The patient was switched to 2 weeks of IP meropenem and was allowed to go home with improving parameters. She was readmitted on day 22 day for culture-negative PD peritonitis that we suspected was due to inadequately cleared Roseomonas gilardii. Despite treatment with multiple IP antibiotics, the catheter was not salvageable.

Ours is the second case of Roseomonas gilardii peritonitis ever reported. Roseomonas is associated with contaminated water source and soil $(1,2)$. Our patient was washing her hands under unhygienic circumstances. Roseomonas peritonitis presents with vague abdominal pain (1-3). Despite 2 weeks of antibiotic treatment, half of the patients in reported cases relapsed within 4 weeks and a third ended up with catheter removal $(3,4)$. Patients with culture-negative peritonitis or with a recent history of Roseomonas infection may benefit from early addition of aminoglycosides, carbapenems, or fluoroquinolones. We therefore suggest IP antibiotic therapy for a minimum of 3 weeks for Roseomonas peritonitis. Relapses must be treated aggressively to avoid refractory peritonitis that will lead to catheter removal and septicemia.

\section{DISCLOSURE}

The authors have no financial conflicts of interest to declare.

\author{
Nephrology Department ${ }^{1}$ \\ Hospital Serdang, Selangor \\ Nephrology Unit ${ }^{2}$ \\ Faculty of Medicine and Health Sciences \\ Universiti Putra Malaysia, Selangor \\ Serdang, Malaysia \\ *email:drchrislim@gmail.com
}

1. Sandoe JA, Malnick H, Loudon KW. A case of peritonitis caused by Roseomonas gilardii in a patient undergoing continuous ambulatory peritoneal dialysis. J Clin Microbiol 1997; 35:2150-2.

2. Bibashi E, Sofianou D, Kontopoulou K, Mitsopoulos E, Kokolina E. Peritonitis due to Roseomonas fauriae in a patient undergoing continuous ambulatory peritoneal dialysis. J Clin Microbiol 2000; 38:456-7.

3. Boyd MA, Laurens MB, Fiorella PD, Medley SR. Peritonitis and technique failure caused by Roseomonas mucosa in an adolescent infected with HIV on continuous cycling peritoneal dialysis. J Clin Microbiol 2012; 50(11):3801-4.

4. Tsai SF, Chen $\mathrm{CH}$, Shu KH, Wu MJ. Peritonitis caused by Roseomonas in a patient undergoing automated peritoneal dialysis: case report and literature review. Intern Med 2012; 51(13):1721-4.

http://dx.doi.org/10.3747/pdi.2016.00103

$$
\begin{aligned}
& \text { S. Malini }{ }^{1} \\
& \text { B.L. Goh } \\
& \text { T.S. Lim } \\
& \text { 2* }
\end{aligned}
$$

This single copy is for your personal, non-commercial use only. For permission to reprint multiple copies or to order presentation-ready copies for distribution, contact Multimed Inc. at marketing@multi-med.com 